\title{
Saúde mental de acadêmicos de medicina: estudo longitudinal
}

\author{
Mental health in medical students: longitudinal study
}

\author{
Mirna Rossi Barbosa-Medeiros' (1) | mirnarossi@hotmail.com \\ Antonio Prates Caldeira' (D) antonio.caldeira@unimontes.br
}

\section{RESUMO}

Introdução: Poucos estudos avaliam de forma longitudinal a saúde mental e qualidade de vida de estudantes de medicina.

Objetivo: Este estudo teve o objetivo de comparar os escores dos sintomas de transtornos psiquiátricos em acadêmicos de medicina ao longo de três anos da graduação, discutindo o contexto da saúde mental dos estudantes longitudinalmente durante o processo de formação.

Método: Trata-se de um estudo longitudinal iniciado em 2015, com estudantes que estavam frequentando o $1^{\circ}$ e o $7^{\circ}$ períodos de graduação de três escolas médicas do norte de Minas Gerais. As mesmas turmas também foram abordadas nos anos seguintes, quando estavam no $3^{\circ}$ e $9^{\circ}$, e no $5^{\circ}$ e $11^{\circ}$ períodos. Foram avaliados os sintomas depressivos, nível de sonolência diurna, nível de saúde geral, dimensões da Síndrome de Burnout, e qualidade de vida. Utilizou-se o teste não-paramétrico de Kruskal Wallis para comparar os três anos da graduação.

Resultados: Dos 248 acadêmicos matriculados nos períodos selecionados, participaram 162 em 2015, 209 em 2016 e 221 em 2017 . Para as turmas iniciantes, os escores do Questionário de Saúde Geral, que indica a presença de Transtornos Mentais Comuns, aumentaram entre 2015 e 2017. No mesmo período, houve aumento dos escores da dimensão descrença e redução significativa nos escores da dimensão eficácia profissional, do Maslach Burnout Inventory, denotando piora na saúde mental para esse grupo. Entre as turmas avaliadas a partir do meio do curso, observa-se aumento significativo nos escores do Questionário de Saúde Geral e na dimensão exaustão emocional do Maslach Burnout Inventory. A sonolência diurna excessiva apresentou oscilações durante os períodos.

Conclusões: Os resultados refletem um agravamento na saúde mental destes estudantes ao longo do curso, especialmente entre o ano de 2015 e 2017, em relação a Transtornos Mentais Comuns e esgotamento profissional. Este resultado chama a atenção para a necessidade de se adotar estratégias que levem o estudante a lidar com os fatores estressantes inerentes ao curso, como o incentivo ao esporte, suporte psicológico, e a reorganização da estrutura curricular do curso, com períodos livres destinados a lazer.

Palavras-chave: Estudantes de Medicina; Depressão; Burnout; Qualidade de Vida.

\section{ABSTRACT}

Introduction: Few studies have longitudinally evaluated the mental health and quality of life of medical students.

Objective: This study aimed to compare the scores of symptoms of psychiatric disorders in medical students over three years of graduation, discussing the context of students' mental health longitudinally during the training process.

Method: This is a longitudinal which study started in 2015, with students who were attending the 1st and 7th undergraduate courses of the three medical schools in the north of Minas Gerais. The same classes were also approached in the following years, when they were in the 3rd and 9th, and in the 5th and 11th periods. Depressive symptoms, daytime sleepiness level, general health level, Burnout Syndrome dimensions, and quality of life were evaluated. The nonparametric Kruskal Wallis test was used to compare the three years of graduation.

Results: Of the 248 students enrolled in the selected periods, 162 participated in 2015, 209 in 2016 and 221 in 2017 . For beginner classes, the scores on the General Health Questionnaire, which indicates the presence of Common Mental Disorders, increased between 2015 and 2017. In the same period, there was an increase in the scores for disbelief dimension and a significant reduction in the scores for the professional efficacy dimension, from the Maslach Burnout Inventory, denoting worsening in mental health for this group. Among the classes evaluated from the middle of the course, there is a significant increase in the scores of the General Health Questionnaire and in the emotional exhaustion dimension of the Maslach Burnout Inventory. Excessive daytime sleepiness presented oscillations during the periods.

Conclusions: The results reflect a worsening in the mental health of these students throughout the course, especially between 2015 and 2017 , in relation to Common Mental Disorders and professional exhaustion. This result calls attention to the need to adopt strategies that lead the student to deal with the stress factors inherent to the course, such as encouraging sports, psychological support, and the reorganization of the curricular structure of the course, with free periods for leisure.

Keywords: Medical Students; Depression; Burnout; Quality of Life.

${ }^{1}$ Universidade Estadual de Montes Claros, Montes Claros, Minas Gerais, Brasil.

Editora-chefe: Daniela Chiesa

Editor associado: Maurício Abreu Pinto Peixoto

Recebido em 17/10/19; Aceito em 16/07/21.

Avaliado pelo processo de double blind review. 


\section{INTRODUÇÃO}

Estudantes de medicina têm sido alvo de diversos estudos que avaliam sua saúde mental e qualidade de vida, devido às grandes pressões vivenciadas durante o curso ${ }^{1-5}$. Esses estudantes apresentam maior prevalência de transtornos tais como a síndrome de Burnout; a população geral ${ }^{6}$, e quando comparados a universitários de outras áreas, exibem maior prevalência de depressão ${ }^{7}$ e distúrbios do sono ${ }^{8}$. A qualidade de vida entre estudantes de medicina também é pior em relação a de indivíduos da mesma faixa etária9.

A partir de metanálise, a literatura nacional registrou prevalência agregada de 30,6\% de sintomas depressivos, $31,5 \%$ de Transtornos Mentais Comuns, 13,1\% de Síndrome de Burnout, $32,9 \%$ de ansiedade, $49,9 \%$ de estresse e $41,6 \%$ de sonolência diurna excessiva em estudantes de medicina. De modo similar, os estudos internacionais também apresentam índices expressivos de transtornos, com prevalência global de depressão de $28,0 \%{ }^{4}$, ansiedade entre $7,7 \%$ e $65,5 \%$ e estresse psicológico variando de $12,2 \%$ a até $96,7 \%$ em estudantes de países de língua inglesa fora da América do Norte $^{2}$.

Algumas queixas são comuns entre os estudantes, como a elevada carga horária de estudos, o cansaço físico, além do desgaste devido ao contato com pacientes terminais e com a morte: aspectos que podem influenciar no surgimento do estresse $^{10}$. Devido à rotina de dormir tarde e levantar cedo, os estudantes de medicina acabam desenvolvendo problemas com sono, fator que também contribui para um prejuízo na saúde mental ${ }^{11}$. Os transtornos que afetam a saúde mental do estudante merecem especial atenção, tendo em vista que alguns deles estão associados a ideações suicidas ${ }^{12}$.

Apesar dos vários estudos sobre a prevalência de transtornos mentais entre estudantes de medicina, a literatura ainda carece de estudos longitudinais que permitam avaliar o comportamento desses transtornos ao longo do curso. É importante considerar ainda o contexto de abertura de várias escolas médicas no Brasil, nos últimos anos ${ }^{13}$. Adicionalmente, a influência da família e a crise econômica pela qual o país tem passado na última década também podem levar alguns acadêmicos a optarem por cursar uma graduação pela qual não têm vocação, com o propósito de garantir uma boa condição de vida no futuro, e as frustrações com o curso podem surgir diante da dificuldade em conciliar a ilusão construída com a realidade vivenciada ${ }^{10}$. Todos esses aspectos tornam os transtornos mentais dos estudantes de medicina uma questão extra de atenção à saúde no ambiente acadêmico. Este estudo teve o objetivo de comparar os escores dos sintomas de transtornos psiquiátricos em acadêmicos do curso médico ao longo de três anos da graduação, discutindo o contexto da saúde mental dos estudantes longitudinalmente durante o processo de formação.

\section{MÉTODOS}

Trata-se de um estudo longitudinal observacional, prospectivo, iniciado em 2015, com estudantes que estavam frequentando $\circ 1^{\circ}$ e $\circ 7^{\circ}$ períodos de graduação das três escolas médicas do norte de Minas Gerais, que representavam - respectivamente - o período inicial e o intermediário do curso. As três escolas médicas ficam localizadas no município de Montes Claros, têm como metodologia de ensino a Aprendizagem Baseada em Problemas (ABP). Uma instituição é pública e duas privadas.

Foram considerados elegíveis para o estudo todos os acadêmicos das turmas selecionadas, desde que regularmente matriculados. As turmas selecionadas foram acompanhadas anualmente em 2015, 2016 e 2017, sendo que a aplicação dos instrumentos de coleta de dados aconteceu no último bimestre de cada ano. As turmas iniciais foram avaliadas, portanto, quando estavam no $1^{\circ}, 3^{\circ}$ e $5^{\circ}$ períodos, enquanto as demais foram avaliadas quando estavam no $7^{\circ}, 9^{\circ}$ e $11^{\circ}$ períodos. Na primeira coleta de dados, não houve grande adesão dos estudantes tendo essa aumentado nas coletas seguintes. Tendo em vista que o estudo não corresponde a uma coorte de indivíduos e sim, de turmas, o número de participantes em cada ano variou. $A$ taxa de participação dos estudantes para 2015, 2016 e 2017 foram respectivamente - de $65,3 \%, 84,3 \%$ e $85,6 \%$.

Os instrumentos de coleta de dados em todas as abordagens foram: Questionário de Saúde Geral (QSG-12)14, Maslach Burnout Inventory - Student Survey (MBI-SS) ${ }^{15}$, Inventário de Depressão de Beck (BDI) ${ }^{16}$, Escala de sonolência diurna de Epworth ${ }^{17}$, Questionário de avaliação da qualidade de vida Short Form 12 (SF-12) ${ }^{18}$, além de questionário de caracterização demográfica e social (sexo, idade, estado civil, instituição onde estuda, com quem reside, renda).

O QSG-12 tem o objetivo de identificar a presença de Transtornos Mentais Comuns. É composto por 12 questões, que incluem problemas com sono e apetite, experiências subjetivas de estresse, tensão ou tristeza, domínio de problemas diários, tomada de decisão, e autoestima, sendo seis questões positivas e seis negativas, com quatro opções de resposta que variam de "absolutamente não" a "muito mais do que de costume". As questões positivas foram invertidas; para cada uma foi usado o escore 0-0-1-1, de forma que o instrumento possui escore total de 0 a 12 pontos $^{19}$. A presença de Transtornos Mentais Comuns está relacionada a escores mais altos no QSG-12. O instrumento foi validado no Brasil, e sua estrutura unifatorial apresenta adequada consistência ${ }^{14}$.

O MBI-SS é um questionário, destinado a avaliar a Síndrome de Burnout em estudantes, com três dimensões: exaustão emocional (5 questões), descrença (4 questões) e eficácia profissional (6 questões). Cada questão possui uma 
escala de 7 pontos que varia de 0 - nunca a 6 - todos os dias. Altos escores em exaustão emocional e descrença e baixos escores em eficácia profissional constituem a Síndrome de Burnout $^{15}$. O instrumento pode ser utilizado com abordagem bi ou tridimensional, mas não possui padronização quanto aos pontos de corte para cada dimensão. No presente estudo, cada uma das dimensões foi avaliada como variável contínua.

O Inventário de Depressão de Beck é uma escala autoaplicada, validada por Gorenstein e Andrade ${ }^{16}$, composta por 21 itens que avaliam os sintomas depressivos presentes na última semana. Cada item possui quatro opções de resposta, que varia de zero a três pontos, podendo obter uma pontuação máxima de 63 pontos. Os escores podem ser classificados em: ausência ou depressão mínima (0 a 9), depressão leve (10 a 18), depressão moderada (18 a 29), e depressão grave (30 a 63). No presente estudo, essa variável foi avaliada de forma numérica.

A escala de sonolência diurna de Epworth tem o propósito de investigar a probabilidade que o indivíduo tem de cochilar em oito situações do dia. Cada questão possui quatro opções de resposta em que zero significa nenhuma chance de cochilar e três significa alta chance de cochilar. A escala atinge valor máximo de 24 pontos. Escores entre 11 e 15 indicam níveis patológicos de sonolência diurna e escores acima de 15 correspondem a níveis muito patológicos ${ }^{17}$.

Para avaliar a qualidade de vida relacionada à saúde, foi aplicado o instrumento SF-12, que contém 12 perguntas, e é dividido em dois domínios: componente físico e componente mental. Cada domínio é transformado em uma escala de 0 a 100 pontos, sendo que os maiores escores indicam melhor qualidade de vida ${ }^{18}$.

A coleta de dados foi realizada na faculdade de origem de cada estudante, no intervalo de suas atividades. Aqueles que aceitaram participar do estudo, assinando o Termo de Consentimento Livre e Esclarecido, preencheram os questionários de forma autoaplicável. Foram consideradas perdas os estudantes que se recusaram a participação no estudo e os que não foram localizados em suas instituições após três tentativas, em dias e horários diferentes.

Os dados foram tabulados e analisados por meio de programa IBM SPSS, versão 19.0. Foram utilizados cálculos de frequência absoluta e relativa, média e desvio-padrão para descrição dos participantes em cada ano do estudo. Para comparar os escores em cada instrumento relacionado à saúde mental dos estudantes entre as três avaliações da graduação foi utilizado o teste não paramétrico de Kruskal Wallis, adotando nível de $5 \%$ de significância. A escolha do teste não-paramétrico se deu após identificação de que as variáveis não seguiam distribuição normal, avaliadas pelo teste Kolmogorov-Smirnov. Também foram feitos diagramas de dispersão entre as médias dos escores de todas as variáveis investigadas e os períodos do curso, e a partir da relação observada foram conduzidas análises de tendência, com seus respectivos coeficientes de determinação.

Este estudo foi aprovado pelo Comitê de Ética em Pesquisa de uma das instituições participantes, sob o parecer $n^{\circ} 1.196 .370$. Todos os estudantes que consentiram participar do estudo assinaram o Termo de Consentimento Livre e Esclarecido.

\section{RESULTADOS}

Dos 248 acadêmicos matriculados nos períodos selecionados nas três escolas médicas, participaram 162 em 2015, 209 em 2016 e 221 em 2017. A média de idade dos estudantes em 2015 foi de 21,3 ( $\pm 3,7)$ anos, em 2016, de 22,5 $( \pm 4,3)$ anos, e em 2017 foi de 23,9 $( \pm 3,9)$ anos. A maioria dos estudantes era do sexo feminino e solteira. As características sociodemográficas dos respondentes para os três anos do estudo estão disponíveis na Tabela 1.

A Tabela 2 apresenta a média dos escores dos questionários que avaliam a saúde geral, sintomas depressivos, dimensões do Burnout, e os componentes físico e mental da qualidade de vida dos acadêmicos das turmas ingressantes no curso nos três anos do estudo. Houve aumento significativo nos escores do QSG, o que indica a presença de Transtornos Mentais Comuns, e da dimensão descrença do MBI-SS, e redução significativa nos escores da dimensão eficácia profissional.

A Tabela 3 apresenta a média dos escores dos questionários que avaliam a saúde geral, sintomas depressivos, dimensões do Burnout, e os componentes físico e mental da qualidade de vida dos acadêmicos das turmas que foram avaliadas a partir do meio do curso nos três anos do estudo. Observa-se aumento significativo nos escores do QSG, e na dimensão exaustão emocional do MBI-SS. Os escores de descrença também aumentaram, mas a diferença não foi significativa. Em relação à sonolência diurna, houve uma redução nos escores entre $7^{\circ}$ e o $9^{\circ}$ períodos e depois um aumento entre o $9^{\circ}$ e o $11^{\circ}$ períodos.

Os gráficos 1 a 3 apresentam as linhas de tendências dos escores referentes ao questionário de Saúde Geral, sonolência diurna, sintomas depressivos, dimensões da Síndrome de Burnout, e componentes da qualidade de vida e suas respectivas equações para todo o período do curso médico. Existe uma tendência de aumento dos escores de sonolência diurna e de morbidade psicológica. Em relação às dimensões da Síndrome de Burnout, os escores de exaustão emocional e descrença tendem a aumentar. 
Tabela 1. Características sociodemográficas dos acadêmicos de medicina do norte de Minas Gerais, entre 2015 e 2017.

\begin{tabular}{|c|c|c|c|c|c|c|c|}
\hline \multirow{2}{*}{ Variáveis } & \multicolumn{2}{|c|}{2015} & \multicolumn{2}{|c|}{2016} & \multicolumn{2}{|c|}{2017} & \multirow{2}{*}{ p-valor } \\
\hline & $\mathbf{n}$ & (\%) & $\mathrm{N}$ & (\%) & $\mathrm{N}$ & (\%) & \\
\hline Sexo & & & & & & & 0,637 \\
\hline Masculino & 59 & $(36,4)$ & 85 & $(40,7)$ & 90 & $(40,7)$ & \\
\hline Feminino & 103 & $(63,6)$ & 124 & $(59,3)$ & 131 & $(59,3)$ & \\
\hline Estado civil & & & & & & & 0,847 \\
\hline Solteiro & 148 & $(91,4)$ & 189 & $(90,5)$ & 195 & $(88,2)$ & \\
\hline Casado / união estável & 13 & $(8,0)$ & 17 & $(8,1)$ & 23 & $(10,4)$ & \\
\hline Divorciado ou viúvo & 1 & $(0,6)$ & 3 & $(1,4)$ & 3 & $(1,4)$ & \\
\hline Tipo de Instituição & & & & & & & 0,081 \\
\hline Pública & 23 & $(14,2)$ & 48 & $(23,0)$ & 38 & $(17,2)$ & \\
\hline Privada & 139 & $(85,8)$ & 161 & $(77,0)$ & 183 & $(82,8)$ & \\
\hline Com quem reside atualmente & & & & & & & 0,915 \\
\hline Sozinho & 19 & $(11,7)$ & 34 & $(16,3)$ & 38 & $(17,2)$ & \\
\hline Com os pais & 58 & $(35,8)$ & 71 & $(34,0)$ & 74 & $(33,5)$ & \\
\hline Com familiares & 35 & $(21,6)$ & 42 & $(20,1)$ & 44 & $(19,9)$ & \\
\hline Com outros estudantes & 39 & $(24,0)$ & 52 & $(24,9)$ & 50 & $(22,6)$ & \\
\hline Outros & 10 & $(6,2)$ & 10 & $(4,8)$ & 15 & $(6,8)$ & \\
\hline Renda & & & & & & & 0,612 \\
\hline Até 3000,00 & 35 & $(21,6)$ & 44 & $(21,1)$ & 48 & $(21,7)$ & \\
\hline Entre 3000,00 e 5500,00 & 36 & $(22,2)$ & 51 & $(24,4)$ & 35 & $(15,8)$ & \\
\hline Entre 5500,00 e 10000,00 & 36 & $(22,2)$ & 53 & $(25,4)$ & 59 & $(26,7)$ & \\
\hline Acima de 10000,00 & 29 & $(17,9)$ & 42 & $(20,1)$ & 44 & $(19,9)$ & \\
\hline Não responderam & 26 & $(16,0)$ & 19 & $(9,1)$ & 35 & $(15,8)$ & \\
\hline
\end{tabular}

(*) Teste Qui-quadrado.

Tabela 2. Média e desvio-padrão dos escores de saúde geral, sonolência diurna, sintomas depressivos, dimensões do Burnout e da qualidade de vida dos acadêmicos comparando o $1^{\circ}, 3^{\circ}$ e $5^{\circ}$ períodos.

\begin{tabular}{lccccccc}
\hline & \multicolumn{2}{c}{$\mathbf{1}^{\circ}$ período } & \multicolumn{2}{c}{$3^{\circ}$ período } & \multicolumn{2}{c}{$\mathbf{5}^{\circ}$ período } & \multirow{2}{*}{ p-valor* } \\
\cline { 2 - 6 } & Média & DP & Média & DP & Média & DP & \\
\hline Questionário de Saúde Geral & 3,75 & 2,62 & 5,60 & 3,35 & 5,76 & 2,96 & $<0,001$ \\
Sonolência diurna & 9,68 & 3,47 & 8,86 & 3,87 & 9,22 & 4,16 & 0,314 \\
Sintomas depressivos & 9,97 & 7,76 & 8,51 & 8,12 & 8,70 & 8,55 & 0,104 \\
Exaustão Emocional & 17,69 & 6,58 & 18,69 & 6,61 & 18,17 & 7,13 & 0,415 \\
Descrença & 6,74 & 6,10 & 8,84 & 6,73 & 9,21 & 6,50 & 0,008 \\
Eficácia Profissional & 28,67 & 6,10 & 26,54 & 6,89 & 25,00 & 7,68 & $<0,001$ \\
PCS - QVRS & 50,67 & 6,96 & 51,67 & 6,74 & 52,51 & 6,65 & 0,087 \\
MCS - QVRS & 43,10 & 10,46 & 42,21 & 10,94 & 41,67 & 10,60 & 0,556 \\
\hline
\end{tabular}

(*) Teste não-paramétrico de Kruskal Wallis. 
Tabela 3.Média e desvio-padrão dos escores de saúde geral, sonolência diurna, sintomas depressivos, dimensões do Burnout e da qualidade de vida dos acadêmicos comparando o $7^{\circ}, 9^{\circ}$ e $11^{\circ}$ períodos.

\begin{tabular}{lccccccc}
\hline & \multicolumn{2}{c}{$\mathbf{7}^{\mathbf{0}}$ período } & \multicolumn{2}{c}{$\mathbf{9}^{\circ}$ período } & \multicolumn{2}{c}{$\mathbf{1 1}^{\circ}$ período } & \multirow{2}{*}{ p-valor* } \\
\cline { 2 - 6 } & Média & DP & Média & DP & Média & DP & \\
\hline Questionário de Saúde Geral & 3,40 & 2,77 & 5,22 & 3,07 & 6,10 & 3,14 & $<0,001$ \\
Sonolência diurna & 10,12 & 4,17 & 8,75 & 4,00 & 10,76 & 4,60 & 0,033 \\
Sintomas depressivos & 9,38 & 8,32 & 7,65 & 7,32 & 9,80 & 7,27 & 0,116 \\
Exaustão Emocional & 18,57 & 6,12 & 18,76 & 6,26 & 21,43 & 6,39 & 0,002 \\
Descrença & 8,12 & 6,49 & 8,54 & 6,48 & 10,20 & 6,53 & 0,099 \\
Eficácia Profissional & 26,77 & 5,93 & 27,09 & 5,95 & 26,69 & 6,13 & 0,923 \\
PCS - QVRS & 49,92 & 7,95 & 51,59 & 6,32 & 50,41 & 6,75 & 0,424 \\
MCS - QVRS & 43,02 & 10,41 & 43,37 & 10,86 & 40,29 & 11,60 & 0,206 \\
\hline
\end{tabular}

$\left.{ }^{*}\right)$ Teste não-paramétrico de Kruskal Wallis.

Gráfico 1. Tendência dos escores de saúde geral, sonolência diurna e sintomas depressivos dos estudantes de medicina ao longo do curso.

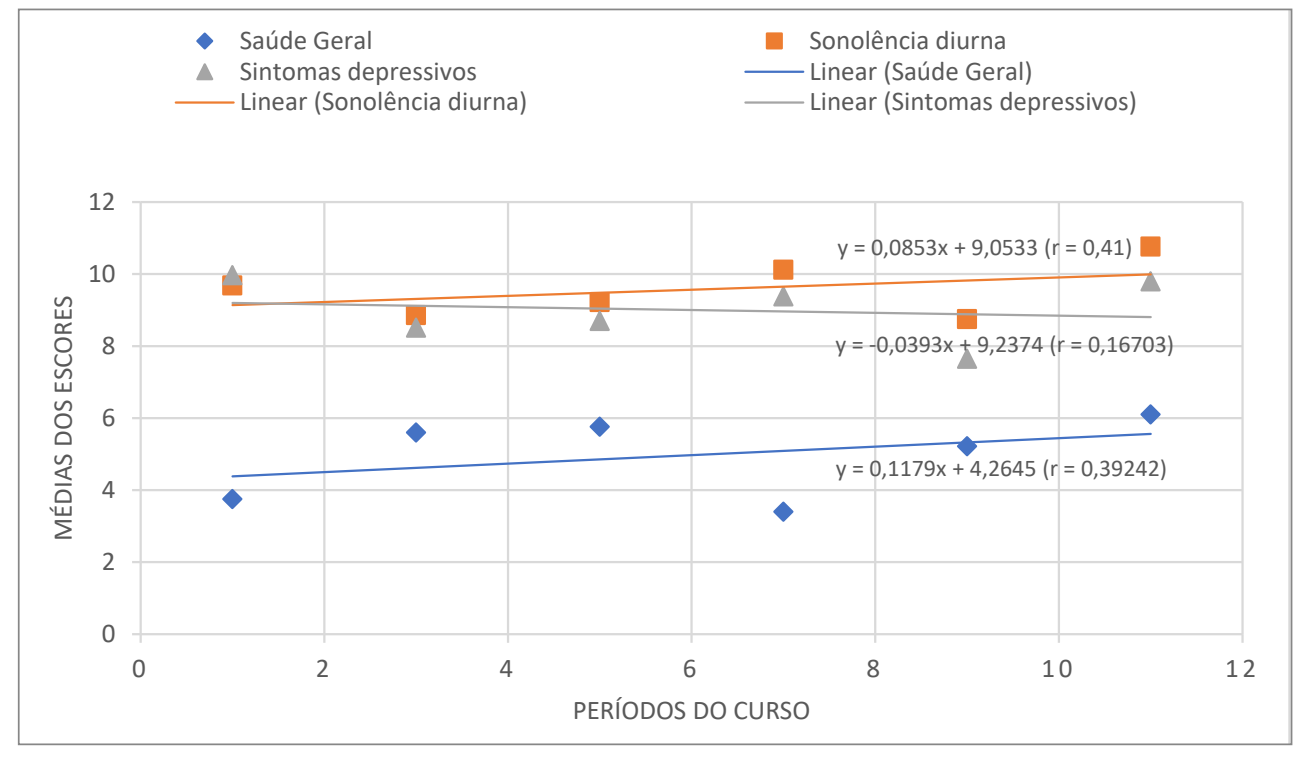

Gráfico 2. Tendência dos escores de exaustão emocional, descrença e eficácia profissional dos estudantes de medicina ao longo do curso.

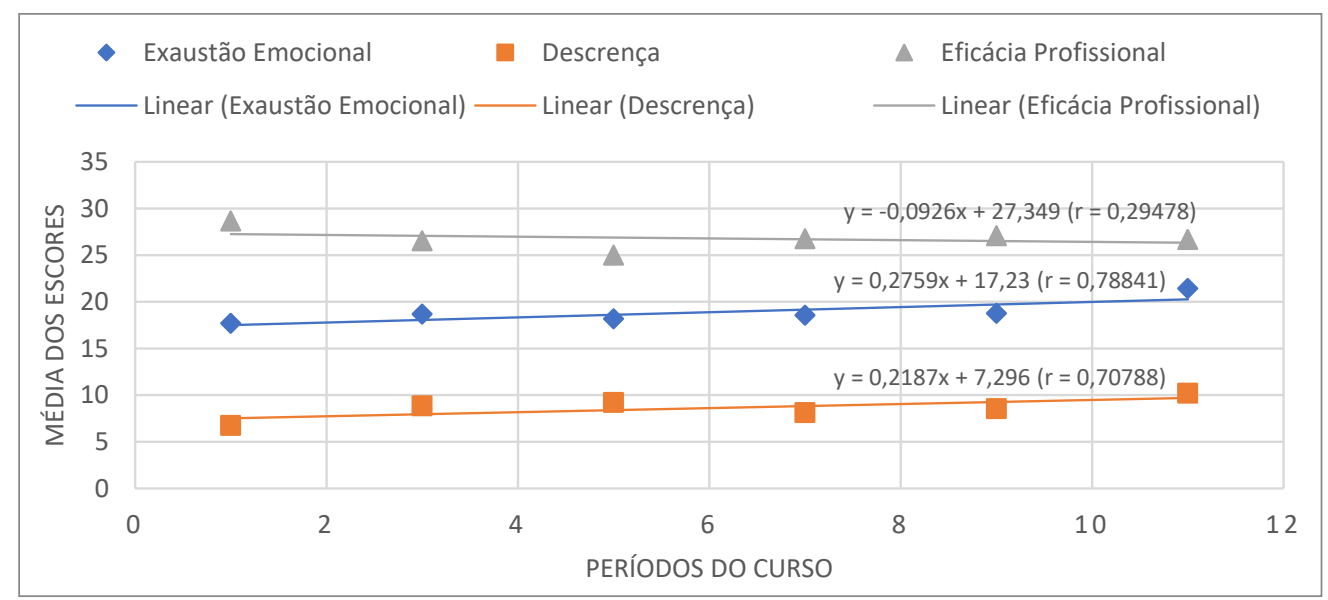


Gráfico 3. Tendência dos escores dos componentes físico e mental da qualidade de vida dos estudantes de medicina ao longo do curso.

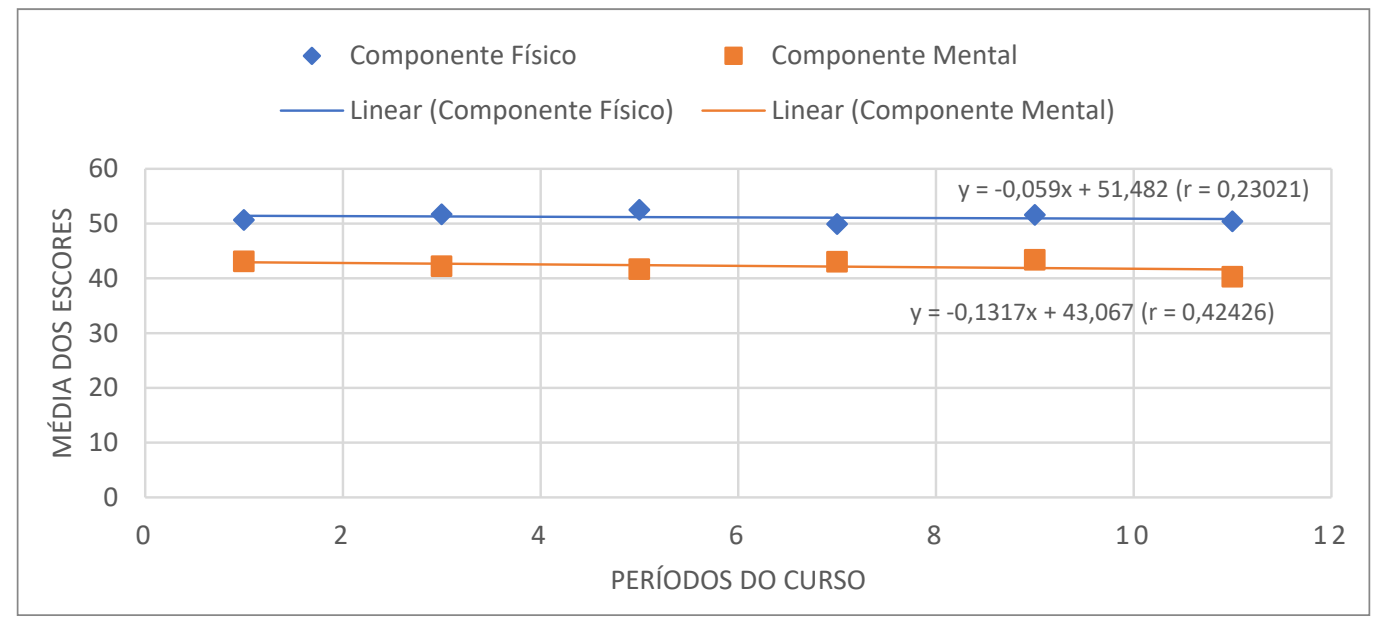

\section{DISCUSSÃO}

Este estudo permitiu avaliar o comportamento da saúde mental dos estudantes das três escolas médicas do norte de Minas Gerais - durante três anos de graduação - e traz à tona novamente o debate sobre a saúde mental dos estudantes em um momento de importantes mudanças na área da saúde e na estrutura da formação médica, considerando a publicação de novas Diretrizes Curriculares Nacionais para os cursos de medicina, no ano de 2014. Foi possível observar uma tendência de aumento nos escores do questionário de saúde geral, indicativo de Transtornos Mentais Comuns, conforme o avanço da graduação. Também houve tendência de aumento nos escores de exaustão emocional e descrença ao longo do curso, o que, de um modo geral, reflete um agravamento na saúde mental destes estudantes. Isso demonstra que eles passaram a se sentir mais estressados, esgotados e mais descrentes com a carreira médica. Os níveis de descrença foram maiores entre os acadêmicos do $11^{\circ}$ período - resultado particularmente alarmante - uma vez que estes acadêmicos já se encontram em fase de internato, e altos níveis de descrença denotam um distanciamento nas relações interpessoais e baixa expectativa em relação ao futuro profissional. Altos níveis de exaustão emocional e descrença correspondem à Síndrome de Burnout; embora esta síndrome não se configure como uma entidade nosológica, identificar a sua presença em estudantes é bastante relevante, uma vez que ela está associada ao contexto em que ocorre, ou seja, alerta para a discussão do quanto o ambiente possa ser desencadeador do sofrimento psíquico.

Estudos transversais que buscaram comparar sintomas que afetam a saúde mental entre períodos do curso apresentaram resultados controversos. Em estudo multicêntrico, que englobou 22 escolas médicas, os acadêmicos do $5^{\circ}$ e $6^{\circ}$ ano apresentaram os piores escores de exaustão emocional e descrença ${ }^{20}$. No mesmo sentido, em outro estudo brasileiro, a adição de um semestre no curso predisse um aumento de 0,08 pontos no escore de descrença e uma diminuição de 0,09 no escore de eficácia profissional ${ }^{21}$. No estudo em faculdade privada de Barretos - São Paulo - a presença de Burnout foi mais frequente nos acadêmicos do $1^{\circ}$ ano, quando comparados aos demais. No entanto, estes mesmos autores inferem que esta síndrome é uma condição crônica em que há pouca expectativa de melhora durante toda a graduação ${ }^{22}$.

No presente estudo, os níveis de sonolência também tiveram uma tendência de crescimento. Pode-se inferir que a chance de cochilar durante o dia, em diversas situações, cresceu devido ao aumento nos níveis de cansaço e estresse destes estudantes. Outros estudos registram índices preocupantes de distúrbios do sono em estudantes de medicina, com sonolência diurna excessiva, mas sem diferenças entre os anos do curso ${ }^{23-24}$.

Com relação aos sintomas depressivos, não houve diferença nos escores entre os períodos. Outros estudos revelam resultados alarmantes entre os estudantes, em qualquer que seja o período do curso. Ao comparar períodos da graduação, os estudos de Bassols et al. ${ }^{25}$ e Brenneisen Mayer et al. ${ }^{26}$, ambos realizados com estudantes brasileiros, não observaram diferenças na prevalência destes sintomas. Já em metanálise realizada com estudos de diferentes continentes, a prevalência de sintomas depressivos foi significativamente maior no $1^{\circ}$ ano do curso, havendo um declínio nos anos seguintes, até o $5^{\circ}$ ano ${ }^{4}$. Em contrapartida, em estudo de Rezende et al. ${ }^{27}$, quanto mais avançado o período do curso, piores os escores no IDB. Pesquisa com estudantes de Camarões também observou maior chance de apresentar sintomas depressivos naqueles que estavam no ciclo clínico ( $4^{\circ}$ ao $7^{\circ}$ ano) comparados aos que estavam no ciclo pré-clínico ( $1^{\circ}$ ao $3^{\circ}$ ano). Baldassin et al..$^{28}$ sugerem que 
os próprios cursos podem aumentar os sintomas depressivos entre os estudantes.

É possível que as diferenças de resultados entre os estudos que compararam períodos/anos do curso tenham ocorrido em virtude das particularidades da estrutura curricular de cada escola médica. Desta forma, os períodos mais estressantes, com maior sobrecarga de estudos, podem variar entre as instituições. Fatores externos, como diferenças regionais, também podem contribuir para uma melhor ou pior saúde mental.

Entretanto, a literatura é unânime em registrar que os fatores estressantes estão presentes desde o início da graduação, com prevalência expressiva de distúrbios que afetam a saúde

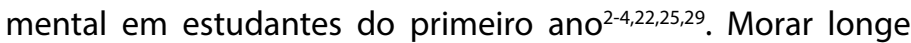
da família e o impacto, ao se deparar com uma metodologia diferente da que estavam habituados, podem ser possíveis razões para este resultado. As exigências dos cursinhos prévestibulares também é outro fator gerador de estresse ${ }^{30}$. Isto significa que os estudantes já ingressam no curso médico com sinais de sofrimento psíquico; por se tratar de uma graduação que exige alto rendimento, estes estudantes tendem a manter altos os níveis de estresse, que podem culminar em outros transtornos mentais, uma vez que o perfil atual da sociedade demonstra uma dificuldade em lidar com situações de pressão. Neste sentido, o curso médico não é unicamente responsável pelo adoecimento dos estudantes, mas um fator que contribui para potencializar o sofrimento psíquico daqueles que já se encontram mais vulneráveis.

Já nos anos seguintes, existem outros fatores que podem ser responsáveis pela presença de estresse, Burnout e outros transtornos entre os estudantes. Pesquisas mostraram que a empatia tem sido um fator protetivo para o surgimento de Burnout, que contribui para a redução dos níveis desta síndrome a partir do segundo ano, mas que devido à característica multifatorial da SB, o comportamento dos estudantes pode sofrer variações positivas ou negativas ${ }^{22}$.

Os resultados observados - de um modo geral-sugerem que há uma aparente melhora nos indicadores de saúde mental, especialmente no estágio intermediário do curso (7º período), e estes indicadores voltam a crescer nos anos seguintes que precedem a conclusão do curso. Todavia, como não se trata da mesma turma acompanhada ao longo dos seis anos, mas sim de duas turmas acompanhadas durante três anos, não se pode descartar a possibilidade de que as turmas avaliadas tenham características distintas, e não se sabe como estava o estado emocional da turma do 70 período nos anos anteriores.

São escassas as pesquisas longitudinais que propuseram avaliar a saúde mental dos estudantes. Estudo brasileiro que acompanhou dezoito acadêmicos do curso médico durante os seis anos da graduação e avaliou as dimensões da SB e o estresse observou que o terceiro e o quarto ano foram considerados os mais difíceis, e que os níveis de descrença aumentam no transcorrer do curso ${ }^{29}$. Estudo que comparou a presença de Transtornos Mentais Comuns entre o início e o final do semestre observou aumento na prevalência deste transtorno ${ }^{31}$. Em contrapartida, outro estudo observou uma estabilidade na prevalência de transtornos mentais ao longo de dois anos da graduação, com discreto aumento nos níveis de estresse e sintomas depressivos na segunda coleta, e melhor qualidade de vida na terceira coleta, o que demonstra a natureza cíclica de tais transtornos, com aumento de sintomatologia para alguns estudantes e redução para outros ${ }^{32}$.

As pesquisas internacionais também confirmam uma piora na saúde mental do estudante ao longo da graduação. Em Melbourne, na Australia, o número de estudantes que relatou sentimentos de angústia psicológica aumentou significativamente no final do ano letivo, comparado ao que sentiram ao início e meio do ano ${ }^{33}$. Estudo longitudinal, com estudantes norte-americanos, registrou um aumento na proporção de estudantes em risco para depressão no terceiro ano quando comparados ao primeiro ano, assim como um aumento no estresse percebido ${ }^{34}$. Na Holanda, um em cada quatro estudantes desenvolveu problemas de saúde mental durante a graduação, em um período de um ano de acompanhamento ${ }^{35}$. Estudantes da Índia foram avaliados para sintomas depressivos, estresse e burnout no primeiro ano da graduação e reavaliados um ano depois. Foi observado aumento significativo nos escores de todos os transtornos, com exceção da dimensão exaustão do Burnout. Os autores concordam que aqueles estudantes que possuem uma leve tendência a um humor depressivo provavelmente sofrerão um agravamento dos sintomas no percurso ${ }^{36}$.

Em relação à qualidade de vida, não houve diferenças significativas em nenhum dos dois componentes. É possível que estes estudantes percebam seu esgotamento acadêmico, mas tenham uma visão particular do que é qualidade de vida, visto que se trata de um conceito abrangente e subjetivo. Além disto, o instrumento utilizado para mensurá-la tem particularidades com ponderações diferenciadas para cada um dos itens avaliados que podem ter influenciado na percepção da qualidade de vida por parte do grupo, mesmo com diferentes níveis de escores nas escalas de QSG, Burnout e sonolência diurna. Por exemplo, o componente mental é mensurado a partir da soma de algoritmos que são acrescidos distintamente para cada um dos itens do questionário, inclusive para os itens sobre função física, aspectos físicos, dor e saúde geral ${ }^{18}$. Neste sentido, como o componente físico demonstrou pequena melhora ao longo dos três anos, é 
possível que o escores nestas questões tenham influenciado na somatória do componente mental.

É importante salientar que - apesar de não ter havido diferença nos componentes da qualidade de vida entre os três anos da graduação - os escores em todos os anos não foram altos e denotam que a qualidade de vida dos estudantes é influenciada negativamente pela graduação, independente do semestre que o acadêmico cursa. Outros estudos também observaram prejuízos na qualidade de vida dos estudantes de medicina ${ }^{9,37}$. Em pesquisa qualitativa realizada com 56 estudantes de seis escolas médicas de várias regiões do Brasil, os estudantes elencaram uma série de fatores que diminuem sua qualidade de vida, que incluem competição, professores despreparados, excesso de atividades, horários que demandam dedicação exclusiva ${ }^{38}$. Estudo longitudinal realizado com estudantes coreanos observou que as mudanças na qualidade de vida são impactadas significativamente pelo suporte social que os estudantes recebem ${ }^{39}$.

De uma forma geral, os resultados encontrados neste estudo chamam a atenção para a necessidade de se adotar estratégias que sejam efetivas no intuito de minimizar os fatores estressantes e o sofrimento emocional dos estudantes. A escola médica se constitui como um estressor por si só, e os estudantes, em geral, têm consciência de que o curso seria cansativo; no entanto, enquanto alguns conseguem lidar naturalmente com tal situação, outros precisam recorrer ao suporte psicológico, devido às características pessoais e aspectos da personalidade que interferem na capacidade que o indivíduo tem de lidar com o estresse ${ }^{40}$.

Uma vezquea empatia está correlacionada positivamente com a eficácia profissional e negativamente com a descrença, ambas dimensões da SB, e que escores mais altos de otimismo estão associados a escores mais altos de eficácia profissional ${ }^{41}$, desenvolver estes sentimentos pode ser uma das alternativas para contribuir na redução do esgotamento profissional entre os estudantes. Em contrapartida, estudo revelou que somente um terço dos acadêmicos com a SB procuraram ajuda ${ }^{42}$. Apesar do fato de que muitas escolas médicas atualmente já contarem com o apoio de profissionais da Psicologia para oferecer este suporte aos estudantes, é preciso repensar as maneiras de garantir a adequado acompanhamento aos estudantes mais necessitados e mesmo adesão dos mesmos às terapias.

Algumas escolas médicas têm reorganizado a estrutura curricular com o objetivo de criar as denominadas "áreas verdes", que consistem em períodos livres destinados a atividades de lazer ${ }^{43}$. Entretanto, os resultados do presente estudo demonstram que as áreas verdes não são suficientes para minimizar o estresse dos estudantes, ou não estão sendo bem aproveitadas. Sabendo que os transtornos apresentados pelos estudantes têm causa multifatorial, as intervenções também devem ser múltiplas ou de caráter mulifacetado. Alguns autores destacam a prática de mindfulness como estratégia capaz de promover o bem-estar mental dos estudantes em ensaio randomizado controlado ${ }^{44}$.

O estudo deve ser considerado à luz de algumas limitações. Não foi possível obter taxa de resposta de $100 \%$ dos estudantes dos períodos selecionados devido às recusas em participar. As escolas estão localizadas em uma região específica do país, e os resultados podem traduzir particularidades dessa região. É importante considerar ainda que alguns resultados - autodeclarados - não traduzam a condição real dos estudantes, que podem se sentir constrangidos a se manifestarem com sofrimento mental, mesmo de forma anônima. Ainda assim, os resultados retratam uma realidade até então desconhecida para a região, que deve servir de alerta aos gestores dos cursos de medicina.

\section{CONCLUSÕES}

Os resultados do presente estudo demonstram uma tendência de aumento nos escores do questionário de saúde geral, indicativo de Transtornos Mentais Comuns, conforme o avanço da graduação. Também houve tendência de aumento nos escores de exaustão emocional e descrença ao longo do curso, com uma aparente melhora no meio do curso, mas que, de um modo geral, reflete um agravamento na saúde mental destes estudantes.

A adoção de estratégias que auxiliem os estudantes a enfrentar situações estressantes devem ser consideradas, como suporte psicológico, projetos esportivos, prática de mindfulness, além da reorganização curricular com os períodos livres durante a semana para o lazer.

\section{CONTRIBUIÇÃO DOS AUTORES}

Mirna Rossi Barbosa-Medeiros contribuiu com a concepção, desenvolvimento do projeto de pesquisa, levantamento da literatura, coleta de dados, análise dos dados, escrita do manuscrito e revisão final. Antônio Prates Caldeira contribuiu com a concepção, desenvolvimento do projeto de pesquisa, análise dos dados, revisão crítica do manuscrito e aprovação da versão final.

\section{CONFLITO DE INTERESSES}

Declaramos não haver conflito de interesses.

\section{FINANCIAMENTO}

Declaramos não haver financiamento. 


\section{REFERÊNCIAS}

1. Pacheco JP, Giacomin HT, Tam WW, Ribeiro TB, Arab C, Bezerra I, et al. Mental health problems among medical students in Brazil: a systematic review and meta-analysis. Rev Bras Psiquiatr. 2017;39(4):369-78.

2. Hope V, Henderson M. Medical student depression, anxiety and distress outside North America: a systematic review. Med Educ. 2014;48(10):963-79.

3. Cuttilan AN, Sayampanathan AA, Ho RC. Mental health issues amongst medical students in Asia: a systematic review [2000-2015]. Ann Transl Med. 2016;4:72.

4. Puthran R, Zhang MW, Tam WW, Ho RC. Prevalence of depression amongst medical students: a meta-analysis. Med Educ. 2016;50:456-68.

5. Serinolli MI, Novaretti MC. A cross-sectional study of sociodemographic factors and their influence on quality of life in medical students at Sao Paulo, Brazil. PLoS ONE. 2017;12(7):e0180009.

6. Dyrbye LN, West CP, Satele D, Boone S, Tan L, Sloan J, et al. Burnout among U.S. medical students, residents, and early career physicians relative to the general U.S. population. Acad Med. 2014;89:443-51.

7. Lei XY, Xiao LM, Liu YN, Li YM. Prevalence of depression among chinese university students: a meta-analysis. PLoS One. 2016;11:e0153454.

8. Li L, Wang YY, Wang SB, Zhang L, Li L, Xu DD, et al. Prevalence of sleep disturbances in Chinese university students: a comprehensive metaanalysis. J Sleep Res. 2018;27(3):e12648.

9. Pagnin D, de Queiroz V. Comparison of quality of life between medical students and young general populations. Educ Health (Abingdon). 2015;28:209-12.

10. Moreira SNT, Silva CAN, Tertulino FF, Tertulino FMF, Vilar MJP, Azevedo GD. Processo de significação de estudantes do curso de Medicina diante da escolha profissional e das experiências vividas no cotidiano acadêmico. Rev Bras Educ Med. 2006;30(2):14-9.

11. Azad MC, Fraser K, Rumana N, Abdullah AF, Shahana N, Hanly PJ, et al. Sleep disturbances among medical students: a global perspective. J Clin Sleep Med. 2015;11:69-74.

12. Torres AR, Campos LM, Lima MCP, Ramos-Cerqueira ATA. Suicidal ideation among medical students: prevalence and predictors. J Nerv Ment Dis. 2018;206(3):160-68

13. Lopes AC. A explosão numérica das escolas médicas brasileiras. Educ Med. 2018;19(1):19-24.

14. Borges LO, Argolo JCT. Adaptação e validação de uma escala de bem-estar psicológico para uso em estudos ocupacionais. Aval Psicol. 2002;1:17-27.

15. Schaufeli WB, Martínez IM, Pinto AM, Salanova M, Bakker AB. Burnout and engagement in university students: a cross-national study. J Cross-Cult Psychol. 2002;33(5):464-81.

16. Gorestein C, Andrade L. Inventário de Depressão de Beck: propriedades psicométricas da versão em português. Rev Psiquiatr Clin. 1998;25(5):245-50.

17. Johns MW. A new method for measuring daytime sleepiness: The Epworth Sleepiness Scale. Sleep. 1991;14:540-5.

18. Camelier AA. Avaliação da qualidade de vida relacionada à saúde em pacientes com DPOC: estudo de base populacional com o SF-12 na cidade de São Paulo-SP [tese]. São Paulo: Universidade Federal de São Paulo; 2005.

19. James D, Yates J, Ferguson F. Can the 12-item general health questionnaire be used to identify medical students who might 'struggle' on the medical course? A prospective study on two cohorts. BMC Med Educ. 2013;13:48.

20. Paro HBMS, Silveira PSP, Perotta B, Gannam S, Enns SC, Giaxa RRB, et al. Empathy among medical students: is there a relation with quality of life and Burnout? Plos One. 2014;9(4):e94133.

21. Pagnim D, Queiroz V, Oliveira-Filho MA, Gonzalez NVA, Salgado AET, Oliveira BC, et al. Burnout and career choice motivation in medical students. Med Teach. 2013;35:388-94.

22. Boni RAS, Paiva CE, Oliveira MA, Lucchetti G, Fregnani JHTG, Paiva BSR Burnout among medical students during the first years of undergraduate school: prevalence and associated factors. Plos One. 2018;13(3):e0191746.

23. Cardoso HC, Bueno FCC, Mata JC, Alves APR, Jochims I, Vaz Filho IHR, et al. Avaliação da qualidade do sono em estudantes de Medicina. Rev Bras Educ Med. 2009;33(3):349-55.
24. Kloster MC, Perotta B, Hauer Junior A, Paro HBMS, Tempski P. Sonolência diurna e habilidades sociais em estudantes de medicina. Rev Bras Educ Med. 2013;37(1):103-9.

25. Bassols AM, Okabayashi LS, Silva AB, Carneiro BB, Feijó F, Guimarães GC et al. First- and last-year medical students: is there a difference in the prevalence and intensity of anxiety and depressive symptoms? Braz J Psichiatry. 2014;36:233-40.

26. Mayer FB, Santos IS, Silveira PSP, Lopes MHI, Souza ARND, Campos EP, et al. Factors associated to depression and anxiety in medical students: a multicenter study. BMC Med Educ. 2016;16:282

27. Rezende CHA, Abrão CB, Coelho EP, Passos LBS. Prevalência de sintomas depressivos entre estudantes de medicina, da Universidade Federal de Uberlândia. Rev Bras Educ Med. 2008;32(3):315-23.

28. Baldassin S, Silva N, Alves TCTF, Castaldelli-Maia JM, Bhugra D, NogueiraMartins MCF, et al. Depression in medical students: cluster symptoms and management. J Affect Disorder. 2013;150(1):110-4.

29. Benevides-Pereira AMT, Gonçalves MB. Transtornos emocionais e a formação em medicina: um estudo longitudinal. Rev Bras Educ Med. 2009;33(1):10-23.

30. Santos FS, Maia CRC, Faedo FC, Gomes GPC, Nunes ME, Oliveira MVM Estresse em estudantes de cursos preparatórios e de graduação em medicina. Rev Bras Educ Med. 2017;41(2):194-200.

31. Ferreira CMG, Kluthcovsky ACGC, Cordeiro TMG. Prevalência de Transtornos Mentais Comuns e Fatores Associados em Estudantes de Medicina: um Estudo Comparativo. Rev Bras Educ Med. 2016;40(2):268-77.

32. Moutinho ILD, Lucchetti ALG, Ezequiel OS, Lucchetti G. Mental health and quality of life of Brazilian medical students: incidence, prevalence, and associated factors within two years of follow-up. Psychiatry Res. 2019;274:306-12

33. Dendle C, Baulch J, Pellicano R, Hay M, Lichtwark I, Ayoub S, et al. Medical student psychological distress and academic performance. Med Teach. 2018;40(12):1257-63.

34. Ludwig AB, Burton W, Weingarten J, Milan F, Myers DC, Kligler B Depression and stress amongst undergraduate medical students. BMC Med Educ. 2015;15:141.

35. Borst JM, Frings-Dresen MHW, Sluiter JK. Prevalence and incidence of mental health problems among Dutch medical students and the studyrelated and personal risk factors: a longitudinal study. Int J Adolesc Med Health. 2016;28(4):349-55.

36. Goel AD, Akarte SV, Agrawal SP, Yadav V. Longitudinal assessment of depression, stressm and burnout in medical students. J Neurosci Rural Pract. 2016;7:493-8.

37. Lins L, Carvalho FM, Menezes MS, Porto-Silva L, Damasceno H. Healthrelated quality of life of students from a private medical school in Brazil. Int J Med Educ. 2015;6:149-54.

38. Tempski P, Bellodi PL, Paro HBMS, Enns SC, Martins MA, Achraiber LB What do medical students think about their quality of life? A qualitative study. BMC Med Educ. 2012;12:106.

39. Hwang IC, Park KH, Kim JJ, Yim J, Ko KP, Bae SM, et al. Perceived social support as a determinant of quality of life among medical students: 6-month follow-up study. Acad Psychiatry. 2017;41(2):180-4.

40. Zonta R, Robles ACC, Grosseman S. Estratégias de enfrentamento do estresse desenvolvidas por estudantes de medicina da Universidade Federal de Santa Catarina. Rev Bras Educ Med. 2006;30(30):147-53.

41. Hojat M, Vergare M, Isenberg G, Cohen M, Spandorfer J. Underlying construct of empathy, optimism, and burnout in medical students. Int J Med Educ. 2015;6:12-6.

42. Dyrbye LN, Eacker A, Durning SJ, Brazeau C, Moutier C, Massie FS, et al. The impact of stigma and personal experiences on the help-seeking behaviors of medical students with Burnout. Acad Med. 2015;90(7):961-9.

43. Antonello ICF. Cuidando de cuidadores em formação nas faculdades de medicina. Rev. Bioét. 2006;14(2):159-62.

44. Vibe M, Solhaug I, Tyssen R, Friborg O, Rosenvinge $\mathrm{JH}$, Sørlie $\mathrm{T}$, et al Mindfulness training for stress management: a randomised controlled study of medical and psychology students. BMC Med Educ. 2013;13:107.

This is an Open Access article distributed under the terms of the Creative Commons Attribution License, which permits unrestricted use, distribution, and reproduction in any medium, provided the original work is properly cited. 